\title{
Some Siegel threefolds with a Calabi-Yau model
}

\author{
EBERHARD FREITAG AND RICCARDO SALVATI MANNI
}

\begin{abstract}
We describe some examples of projective Calabi-Yau manifolds which arise as desingularizations of Siegel threefolds. There is a certain explicit product of six theta constants which defines a cusp form of weight three for a certain subgroup of index two of the Hecke group $\Gamma_{2,0}$ [2]. This form defines an invariant differential form for this group and for any subgroup of it. We study the question whether the Satake compactification for such a subgroup admits a projective desingularization on which this differential form is holomorphic and without zeros. Then this desingularization is a Calabi-Yau manifold. We shall prove: For any group between $\Gamma_{2}[2]$ and $\Gamma_{2,0}$ [2] there exists a subgroup of index two which produces a (projective) Calabi-Yau manifold. The proof rests on a detailed study of this cusp form and on Igusa's explicit desingularization of the Siegel threefolds with respect to the principal congruence subgroup of level $q>2$ (we need $q=4$ ). For a particular case we produce the equations for the corresponding Siegel threefold.
\end{abstract}

Mathematics Subject Classification (2010): 11F46 (primary); 14J32 (secondary).

\section{Introduction}

In the following we describe some examples of Calabi-Yau manifolds that arise as desingularizations of certain Siegel threefolds. Here by a Calabi-Yau manifold we understand a smooth complex projective variety which admits a holomorphic differential form of degree three without zeros and such that the first Betti number is zero. This differential form is unique up to a constant factor, and we call it the Calabi-Yau form. Our interest in this subject is influenced by work of Gritsenko and many discussions with him. The first Siegel modular variety with a Calabi-Yau model and the essentially only one up to now has been discovered by Barth and Nieto. They showed that the "Nieto quintic" $\left\{x \in P^{5}(\mathbb{C}), \sigma_{1}(x)=\sigma_{5}(x)=0\right\}$, where $\sigma_{i}$ denote the elementary symmetric polynomials, has a Calabi-Yau model and they derived that the Siegel modular variety $A_{1,3}(2)$ of polarization type $(1,3)$ and a certain level-two structure has a Calabi-Yau model. Since the Jacobian of a symplectic substitution is $\operatorname{det}(C Z+D)^{-3}$, the Calabi-Yau three-form, produces a modular form of weight three and this must be a cusp form, since it survives on a 
non-singular model as a holomorphic differential form [3, III.2.6]. In the paper [5] Gritsenko and Hulek gave a direct construction of this modular form and obtained a new proof for the fact that $A_{1,3}(2)$ has a Calabi-Yau model. We also refer to [8] for further investigations. Besides this example and some small extensions of this group with the same three-form no other examples of Siegel threefolds with Calabi-Yau model seem to be known. Gritsenko raised the problem of determing all Siegel threefolds which admit a Calabi-Yau model. As we mentioned already, such a threefold will produce a certain cusp form of weight three for the considered modular group $\Gamma$. This cusp form has very restrictive properties. Since the induced differential form should have no zero at least at the regular locus of the quotient $\mathbb{H}_{2} / \Gamma$, all zeros of the form must be contained in the ramification of $\mathbb{H}_{2} \rightarrow \mathbb{H}_{2} / \Gamma$. Gritsenko gave examples of such modular forms: we refer to the paper [4] which contains some systematic study of them. One example that Gritsenko and Cléry describe is the form $\nabla_{3}$, which is a cusp form of weight three for the Hecke group $\Gamma_{2,0}[2]$ with respect to a certain quadratic character $\chi$. Hence a subgroup of index two is a candidate for producing a Calabi-Yau manifold. We will prove that this is the case. We will show more: for any group between $\Gamma_{2}[2]$ and $\Gamma_{2,0}[2]$ there exists a subgroup of index two (the kernel of $\chi$ ) which produces a Calabi-Yau manifold.

The modular form $\nabla_{3}$ will come up in a completely different manner. It is simply the product $T$ of 6 (of the 10 classical) theta constants with suitable properties. In this form it has already been described in [7] and these expressions occur also in [6]. This approach has the advantage that we can easily describe the action of the full modular group, which is necessary, since we need information about this form at all boundary components. Another advantage of this description is that we can use the work of Igusa about the structure of the ring of modular forms with respect to his group $\Gamma_{2}[4,8]$ and of some groups containing this group. Igusa used the ten theta constants of the first kind. If one is concerned with groups in the region of the principal congruence subgroup of level two, there are advantages in using the theta constants of the second kind. We use the very nice approach given by Runge $[15,16]$.

We also make use of Igusa's method of desingularization of the Siegel threefold with respect to the principal congruence subgroup of level $q>2$ (we need $q=4$ ).

Using Igusa's results or Runge's approach, it is easy to determine the rings of modular forms for the groups in question, and in this way one can produce equations for the Siegel threefolds. The main example is the subgroup of index two of $\Gamma_{2}[2]$.

In this introduction we only describe the equations of this Siegel threefold in a purely algebraic way.

Theorem 1.1. Let $X$ be the subvariety of $P^{5}(\mathbb{C})$ given by the intersection of the quartic

$$
y_{5}^{4}=y_{0} y_{1} y_{2}\left(y_{0}+y_{1}+y_{2}+y_{3}+y_{4}\right)
$$

and the quadric

$$
2 y_{5}^{2}=y_{0} y_{1}+y_{0} y_{2}+y_{1} y_{2}-y_{3} y_{4} .
$$

This is a normal projective variety of dimension three. There exists a desingularization $\tilde{X} \rightarrow X$ which is a Calabi-Yau manifold. 
The variety $X$ together with the Calabi-Yau form have some symmetries. They are easier to describe in another coordinate system (see Theorem 5.5 for the explicit description). In this coordinate system we also will give an explicit algebraic expression for the Calabi-Yau form. We will see:

Theorem 1.2. There is a subgroup $G \subset P G L(5, \mathbb{C})$, isomorphic to the semidirect product $S_{3} \cdot(\mathbb{Z} / 2 \mathbb{Z})^{2}$, which leaves $X$ and the form $\omega$ invariant. For each subgroup $H$ of $G$ the quotient $X / H$ admits a desingularization which is a Calabi-Yau manifold.

It is not difficult to determine the singular locus of $X$ :

Proposition 1.3. The singular locus of $X$ is the union of 15 smooth curves. It consists of two G-orbits. One orbit consists of three quadrics, the other of 12 lines. Representatives are given by the ideals

$$
\left(y_{0}+y_{4}, y_{1}+y_{4}, y_{3}-y_{4}, y_{2} y_{4}+y_{5}^{2}\right), \quad\left(y_{0}, y_{2}, y_{3}, y_{5}\right) \text {. }
$$

The main problem is to find a good resolution of the singularities of $X$. It might be possible to do this by hand or with the help of a computer. We will find it by interpreting $X$ as a Siegel modular variety.

ACKnOwledgements. We would like to thank T. Bridgeland, A. Corti, S. Cynk and C. Meyer for useful discussions.

\section{The Siegel modular group of genus two}

Recall that the real symplectic group

$$
\operatorname{Sp}(n, \mathbb{R})=\left\{M \in \operatorname{GL}(2 n, \mathbb{R}) ; \quad{ }^{t} M I M=I\right\} \quad\left(I=\left(\begin{array}{cc}
0 & -E \\
E & 0
\end{array}\right)\right)
$$

acts on the generalized half-plane

$$
\mathbb{H}_{n}:=\left\{Z=X+\mathrm{i} Y ; \quad Z={ }^{t} Z, Y>0 \text { (positive definite) }\right\}
$$

by

$$
M Z=(A Z+B)(C Z+D)^{-1}, \quad M=\left(\begin{array}{ll}
A & B \\
C & D
\end{array}\right) .
$$

Let $\Gamma_{n}:=\operatorname{Sp}(n, \mathbb{Z})$ be the Siegel modular group. The principal congruence subgroup of level $l$ iy

$$
\Gamma_{n}[l]:=\operatorname{kernel}(\operatorname{Sp}(n, \mathbb{Z}) \longrightarrow \operatorname{Sp}(n, \mathbb{Z} / l \mathbb{Z}))
$$


and Igusa's subgroup is

$$
\Gamma_{n}[l, 2 l]:=\left\{M \in \Gamma_{n}[l] ; \quad A^{t} B / l \text { and } C^{t} D / l \text { have even diagonal }\right\} .
$$

For even $l$, Igusa's subgroup is a normal subgroup of $\Gamma_{n}$. Other important subgroups are

$$
\Gamma_{n, 0}[l]=\left\{M \in \Gamma_{n} ; \quad C \equiv 0 \bmod l\right\} .
$$

\subsection{Theta characteristics in genus two}

By definition, a theta characteristic is an element $m=\left(\begin{array}{l}a \\ b\end{array}\right)$ from $(\mathbb{Z} / 2 \mathbb{Z})^{2 n}$.

Here $a, b \in(\mathbb{Z} / 2 \mathbb{Z})^{n}$. The characteristic is called even if ${ }^{t} a b=0$. The group $\operatorname{Sp}(n, \mathbb{Z} / 2 \mathbb{Z})$ acts on the set of characteristics by

$$
M\{m\}:={ }^{t} M^{-1} m+\left(\begin{array}{c}
\left(A^{t} B\right)_{0} \\
\left(C^{t} D\right)_{0}
\end{array}\right) .
$$

Here $S_{0}$ denotes the column built of the diagonal of a square matrix $S$. It is wellknown that $\operatorname{Sp}(n, \mathbb{Z} / 2 \mathbb{Z})$ acts transitively on the subsets of even and odd characteristics. Recall that for any characteristic the theta function

$$
\vartheta[m]=\sum_{g \in \mathbb{Z}^{n}} e^{\pi \mathrm{i}\left(Z[g+a / 2]+{ }^{t} b(g+a / 2)\right)}
$$

can be defined. Here we use the identification of $\mathbb{Z} / 2 \mathbb{Z}$ with the subset $\{0,1\} \subset \mathbb{Z}$. It vanishes if and only if $m$ is odd. Recall also that the formula

$$
\vartheta[M\{m\}](M Z)=v(M, m) \sqrt{\operatorname{det}(C Z+D)} \vartheta[m](Z)
$$

holds for $M \in \Gamma_{n}$, where $v(M, m)$ is a rather delicate $8^{\text {th }}$ root of unity which depends on the choice of the square root.

We are interested in the case $n=2$. In this case there are ten even characteristics. We will write the coordinates of $\mathbb{H}_{2}$ as

$$
Z=\left(\begin{array}{ll}
z_{0} & z_{1} \\
z_{1} & z_{2}
\end{array}\right)
$$

A set $\left\{m_{1}, m_{2}, m_{3}, m_{4}\right\}$ of four pairwise different even characteristics is called syzygetic if the sum of any three of them is even. There are 15 syzygetic (unordered) quadruples and the group $\operatorname{Sp}(2, \mathbb{Z})$ acts transitively on them. We call

$$
\left\{\begin{array}{llll}
0 & 0 & 0 & 0 \\
0 & 0 & 0 & 0 \\
0 & 1 & 0 & 1 \\
0 & 0 & 1 & 1
\end{array}\right\}
$$

the standard syzygetic quadruple. We are also interested in the 15 complementary sextuples of even characteristics $\left\{n_{1}, \ldots, n_{6}\right\}$. From a detailed study of the multipliers $v(M, m)$ one can deduce: 
Lemma 2.1. Let $\mathfrak{n}=\left\{n_{1}, \ldots, n_{6}\right\}$ be a sextuple of even characteristics (in the case $n=2$ ) complementary to a syzygetic quadruple. Then the product

$$
T=T_{\mathfrak{n}}:=\prod_{\nu=1}^{6} \vartheta\left[n_{\nu}\right](Z)
$$

is a cusp form of weight 3 for a group conjugate to $\Gamma_{2,0}[2]$ and with respect to a quadratic character $\chi_{\mathfrak{n}}$ on this group. The kernel of this character contains $\Gamma_{2}$ [4]. In the case of the standard tuple the group is $\Gamma_{2,0}[2]$ and the kernel

$$
\Gamma_{2,0}[2]_{\mathfrak{n}}:=\left\{M \in \Gamma_{2,0}[2], \quad \chi_{\mathfrak{n}}(M)=1\right\}
$$

is a subgroup of index two.

We describe the character of $T$ for the standard syzygetic quadruple. For this we introduce

$$
\Theta:=\vartheta\left[\begin{array}{l}
00 \\
01
\end{array}\right] \vartheta\left[\begin{array}{l}
00 \\
00
\end{array}\right] \vartheta\left[\begin{array}{l}
00 \\
10
\end{array}\right] \vartheta\left[\begin{array}{l}
00 \\
11
\end{array}\right] .
$$

Since $T \cdot \Theta=\chi_{5}$ is a modular form with respect to the full modular group, this form also is a modular form for $\Gamma_{2,0}$ [2]. The characters of $T$ and $\Theta$ differ by the character of $\chi_{5}$. Using the well-known isomorphism $\Gamma_{2} / \Gamma_{2}[2] \cong S_{6}$, the character of $\chi_{5}$ corresponds to the sign character of $S_{6}$. Hence it is sufficient to describe the character of $\Theta$.

Lemma 2.2. The character of $\Theta$ for $M \in \Gamma_{2,0}[2]$ is given by

$$
(-1)^{(\alpha+\beta+\gamma) / 2}
$$

where

$$
C^{t} D=\left(\begin{array}{ll}
\alpha & \beta \\
\beta & \gamma
\end{array}\right)
$$

The character of $T$ is the product of this character and the only nontrivial character of the full modular group.

The proof can be taken from [17, Lemma 4].

We have to recall the location of the zeros of the theta functions. In the case ${ }^{t} m=(1,1,1,1)$ the theta function $\vartheta[m]$ has a zero of first order along the diagonal $z_{1}=0$ and every zero component is equivalent to the diagonal $\bmod \Gamma_{2}[1,2]$. We consider the differential form

$$
\omega=\omega_{\mathfrak{n}}=T_{\mathfrak{n}} \mathrm{d} z_{0} \wedge \mathrm{d} z_{1} \wedge \mathrm{d} z_{2}
$$

It is invariant under the group $\Gamma_{2,0}[2]_{\mathfrak{n}}$. 
Proposition 2.3. Let $F \subset \mathbb{H}_{2}$ be an irreducible component of the zero locus of $\omega_{\mathfrak{n}}$. Then there exists an element $M \in \Gamma_{2}$ whose fixed point set is $F$. It has the property $M^{2}=E$. This element is uniquely determined up to the sign and is actually contained already in

$$
\Gamma_{2}[2,4]_{\mathfrak{n}}=\Gamma_{2}[2,4] \cap \Gamma_{2,0}[2]_{\mathfrak{n}} .
$$

Proof. Taking a conjugate group we may assume that $F$ is the diagonal $z_{1}=0$. Then in the sextuple the characteristic ${ }^{t} m=(1,1,1,1)$ must occur. The only non trivial modular substitution which fixes the diagonal is $z_{1} \mapsto-z_{1}$. The theta series for ${ }^{t} m=(1,1,1,1)$ changes its sign under this substitution. The others are invariant. This shows

$$
T_{\mathfrak{n}}\left(\begin{array}{cc}
z_{0} & -z_{1} \\
-z_{1} & z_{2}
\end{array}\right)=-T_{\mathfrak{n}}\left(\begin{array}{ll}
z_{0} & z_{1} \\
z_{1} & z_{2}
\end{array}\right)
$$

Since this is the transformation law for a modular form of odd weight, the substitution $z_{1} \mapsto-z_{1}$ is in the kernel of $\chi_{\mathfrak{n}}$. It is also in $\Gamma_{2}[2,4]$.

We have to consider the group

$$
\Gamma=\Gamma_{\mathfrak{n}}:=\left\{M \in \Gamma_{2}[2] ; \quad \chi_{\mathfrak{n}}(M)=1\right\} .
$$

This is a subgroup of index two of $\Gamma_{2}[2]$. Using Lemma 2.2 and some computation one can see:

Lemma 2.4. For the standard syzygetic quadruple, the group $\Gamma$ is defined inside $\Gamma_{2}[2]$ by the condition that the symmetric matrix $C^{t} D=\left(\begin{array}{ll}\alpha & \beta \\ \beta & \gamma\end{array}\right)$ has the property $\alpha+\beta+\gamma \equiv 0 \bmod 4$. Moreover $\Gamma=\Gamma_{\mathfrak{n}} \subset \Gamma_{2}[2]$ is generated by

1. The group $\Gamma_{2}[4]$.

2. The elements of $\Gamma_{2,0}[2]_{\mathfrak{n}}$ that are conjugate inside $\Gamma_{2}$ to the diagonal matrix with diagonal $(1,-1,1,-1)$.

3. All elements of $\Gamma_{2,0}[2]_{\mathfrak{n}}$ that are conjugate inside $\Gamma_{2}$ to a translation matrix $\left(\begin{array}{ll}E & S \\ 0 & E\end{array}\right)$ of $\Gamma_{2}[2]$.

\section{Igusa's desingularization}

We consider the principal congruence subgroup $\Gamma_{2}[l]$ of level $l \geq 3$ and denote by

$$
X=X(l):=\overline{\mathbb{H}_{2} / \Gamma_{2}[l]}
$$

the Satake compactification and by $\tilde{X}=\tilde{X}(l)$ the monoidal transform along the Satake boundary. Igusa proved that $\tilde{X}$ is smooth. The theory of Igusa is very difficult but fortunately we can formulate in a very simple way what we need from it. 
Igusa used so-called normal coordinates

$$
q_{0}=e^{2 \pi \mathrm{i}\left(z_{0}+z_{1}\right) / l}, \quad q_{2}=e^{2 \pi \mathrm{i}\left(z_{2}+z_{1}\right) / l}, \quad q_{1}=e^{-2 \pi \mathrm{i} z_{1} / l} .
$$

We consider them as a holomorphic map

$$
\mathbb{H}_{2} \longrightarrow \mathbb{C}^{3}, \quad\left(\begin{array}{ll}
z_{0} & z_{1} \\
z_{1} & z_{2}
\end{array}\right) \longmapsto\left(q_{0}, q_{1}, q_{2}\right)
$$

The image is an open subset $D \subset \mathbb{C}^{3}$. If we denote by $\mathcal{T}=\mathcal{T}(l)$ the group of translations $Z \mapsto Z+l S, S$ integral, we get a biholomorphic map

$$
\mathbb{H}_{2} / \mathcal{T} \stackrel{\sim}{\longrightarrow} D
$$

We can consider its inverse map and compose it with the projection onto $\mathbb{H}_{2} / \Gamma_{2}[l]$ and the inclusion into $X$ to get a holomorphic map

$$
D \longrightarrow X
$$

A domain $U \subset \mathbb{C}^{n}$ is called a Reinhardt domain if

$$
\left(z_{1}, \ldots, z_{n}\right) \in U \Longrightarrow\left(\zeta_{1} z_{1}, \ldots, \zeta_{n} z_{n}\right) \in U \quad \text { for }\left|\zeta_{v}\right|=1
$$

It is called a complete Reinhardt domain, if this is true for all $\zeta$ with $\left|\zeta_{v}\right| \leq 1$. Each Reinhardt domain can be completed to a complete Reinhardt domain in an obvious way. Any holomorphic function on a Reinhardt domain can be expanded in a Laurent series in the whole domain. This Laurent series is a power series if and only if the function extends as a holomorphic function to the completed Reinhardt domain.

Lemma 3.1. The domain $D$ is a Reinhardt domain $\mathbb{C}^{3}$. Its completion is

$$
\tilde{D}:=D \cup\left\{q \in \mathbb{C}^{3} ; q_{0} q_{1} q_{2}=0\right\} .
$$

The domain $D$ is dense in $\tilde{D}$.

All we need from Igusa's theory is:

Theorem 3.2 (Igusa). The natural map $D \rightarrow X$ extends to a locally biholomorphic map

$$
\tilde{D} \longrightarrow \tilde{X}
$$

The group $\Gamma_{2} / \Gamma_{2}[l]$ acts on $X$ and hence on $\tilde{X}$. The translates of the images of $\tilde{D}$ cover $\tilde{X}$. 
Each holomorphic function on $D$ can be written as Laurent series in the variables $q_{v}$. Assume that it is the Fourier expansion of a modular form

$$
\sum_{T} a(T) e^{2 \pi \mathrm{i} \sigma\left(t_{0} z_{0}+2 t_{1} z_{1}+t_{2} z_{2}\right) / l}, \quad T=\left(\begin{array}{ll}
t_{0} & t_{1} \\
t_{1} & t_{2}
\end{array}\right) .
$$

Here $T$ runs over all matrices such that $t_{0}, t_{2}, 2 t_{1}$ are integral. If we rewrite this in the normal coordinates, we get

$$
\sum_{T} a(T) q_{0}^{t_{0}} q_{1}^{t_{0}+t_{2}-2 t_{1}} q_{2}^{t_{2}}
$$

By Koecher's principle we can have $a(T) \neq 0$ only for semi-positive $T$. This means $t_{0}, t_{2} \geq 0$ and $t_{0} t_{2} \geq t_{1}^{2}$. This implies $t_{0}+t_{2}-2 t_{1} \geq 0$. Hence the Laurent series actually is a power series. Hence this function extends to a holomorphic function on the whole $\tilde{D}$.

We can talk about the multiplicity of the zero along each of the $q_{v}=0$. The theta functions $\vartheta[m]$ are periodic under $Z \mapsto 8 S, S$ integral. Hence we can use $l=8$ for the normal coordinates. The multiplicities are easy to compute:

Lemma 3.3. The multiplicity of $\vartheta[m]$ along $q_{v}=0$ in the coordinates

$$
q_{0}=e^{2 \pi \mathrm{i}\left(z_{0}+z_{1}\right) / 8}, \quad q_{2}=e^{2 \pi \mathrm{i}\left(z_{2}+z_{1}\right) / 8}, \quad q_{1}=e^{-2 \pi \mathrm{i} z_{1} / 8},
$$

is

$$
a_{1}, \quad a_{2}, \quad a_{1}+a_{2}-2 a_{1} a_{2}, \quad m=\left(\begin{array}{l}
a \\
b
\end{array}\right) .
$$

Let $m_{1}, \ldots, m_{4}$ be a syzygetic quadruple of even characteristics and let $\mathfrak{n}=$ $\left\{n_{1}, \ldots, n_{6}\right\}$ be the complementary even characteristics. We introduced the modular form

$$
T=T_{\mathfrak{n}}=\vartheta\left[n_{1}\right] \cdots \vartheta\left[n_{6}\right] .
$$

By Lemma 2.1 this is a cusp form of weight three for a conjugate of the group $\Gamma_{0}[2]$. Its character is trivial on $\Gamma_{2}[4]$. Hence it is better now to use $l=4$, i.e. to change the notation and to use the normal coordinates

$$
q_{0}=e^{2 \pi \mathrm{i}\left(z_{0}+z_{1}\right) / 4}, \quad q_{2}=e^{2 \pi \mathrm{i}\left(z_{2}+z_{1}\right) / 4}, \quad q_{1}=e^{-2 \pi \mathrm{i} z_{1} / 4} .
$$

We can consider the differential form

$$
\omega=T \mathrm{~d} z_{0} \wedge \mathrm{d} z_{1} \wedge \mathrm{d} z_{2}
$$

on $\tilde{X}(4)$. For general reasons this gives a regular differential form on the whole $\tilde{X}(4)$. We are interested in the zero divisor of this form. We pull it back to $\tilde{D}$ to obtain

$$
C \frac{T}{q_{0} q_{1} q_{2}} \mathrm{~d} q_{0} \mathrm{~d} q_{1} \mathrm{~d} q_{2}
$$


At the moment we only are interested in the behavior of the differential forms along the three divisors $q_{v}=0$. From Lemma 3.3 we can read off the vanishing order of $T$ along $q_{v}=0$. Of course the result depends on the choice of the syzygetic quadruple. For example the vanishing order of the power series along $q_{0}=0$ is given by

$$
\frac{a_{11}+\cdots+a_{61}}{2}, \quad n_{v}=\left(\begin{array}{l}
a_{v} \\
b_{v}
\end{array}\right) .
$$

(The denominator 2 occurs since we used different $q_{v}$ in Lemma 3.3.) By inspection of the 15 cases one sees that this expression is always 1 or 2 . Since for the order of differential form one has to subtract 1 , one gets for the vanishing order of the differential form along $q_{0}$ either 0 or 1 . The same argument works for the variables $q_{1}$ and $q_{2}$. Now one has to use a list of all the 15 syzygetic quadruples. By inspection one finds:

Proposition 3.4. There are 15 syzygetic quadruples. We denote the vanishing order of the differential form $\omega$ (pulled back to $\tilde{D}$ ) at $q_{v}=0$ by $k_{v}$. Then one has for $\left(k_{0}, k_{1}, k_{2}\right)$ the possibilities

$$
\begin{array}{ll}
(0,0,0) & \text { (eight cases), } \\
(1,1,1) & \text { (one case) } \\
(0,0,1) & \text { (two cases), } \\
(0,1,0) & \text { (two cases), } \\
(1,0,0) & \text { (two cases) }
\end{array}
$$

Let us assume that the order along $q_{0}=0$ is one. Then we are in the case $a_{11}+\cdots+$ $a_{61}=4$. A glance at the power series shows that then only even $t_{0}$ occur. Hence the series is invariant under the transformation $q_{0} \mapsto-q_{0}$. This transformation is induced by the translation $z_{0} \mapsto z_{0}+2$. Hence this translation belongs to our group $\Gamma_{\mathfrak{n}}$. This translation acts on $\tilde{X}(4)$ as a reflection, meaning that it is of order 2 and fixes an irreducible subvariety of codimension 1 . So we obtain the following result which extends Proposition 2.3 to the boundary:

Proposition 3.5. The modular form $T$ induces on $\tilde{X}(4)$ a differential form with the following property: if $F \subset \tilde{X}$ is an irreducible component of its zero divisor then there exists a reflection in $\Gamma / \pm \Gamma_{2}[4]$, with fixed point set $F$. The multiplicities of the zeros are one.

We need the following general result:

Theorem 3.6. Let $M$ be a quasi-projective smooth variety of dimension three and $G$ a finite group of automorphisms of it. Assume that every point of $M / G$ admits an open neighborhood such that on its regular locus exists a three-form without zeros. Then $M / G$ admits a quasi-projective crepant ${ }^{1}$ desingularization.

1 "Crepant" means that the inverse images of these three-forms have no poles or zeros at the exceptional divisors [13]. 
Proof. This theorem is a consequence of [2, Theorem 1.2]. Unfortunately the assumptions are slightly different from ours. This theorem only directly applies if an arbitrary stabilizer $G_{a}$ acts on the tangent space as a subgroup of SL().

For this reason we want to give some details. A cluster is a zero dimensional closed subscheme of $M$. The set of all clusters of a fixed degree is parametrized by a quasi-projective Hilbert scheme. The set of all $G$-invariant clusters of degree \# $G$ is a closed subscheme. We denote by $G-\operatorname{Hilb}(M)$ the irreducible component which contains the generic clusters (given by $\# G$ distinct points.) Then

$$
G-\operatorname{Hilb}(M) \longrightarrow M
$$

is a crepant resolution. This can be proved analytically locally. Therefore we can assume that $M=\mathbb{C}^{3}$ and $G \subset \operatorname{GL}(n, \mathbb{C})$ is a linear group. Consider the subgroup $G^{\prime} \in G$ generated by all pseudo-reflections. By a result of Chevalley $\mathbb{C}^{3} / G^{\prime}$ can be identified with $\mathbb{C}^{3}$ in such a way that $H=G / G^{\prime}$ acts linearly. Now our assumption gives that $H$ acts by a subgroup of SL(). Hence we can apply the above mentioned theorem 1.2 in [2] and obtain that

$$
H-\operatorname{Hilb}\left(\mathbb{C}^{3} / G^{\prime}\right) \longrightarrow \mathbb{C}^{3} / G
$$

is a crepant resolution. Taking inverse images one obtains a natural closed embedding

$$
H \text {-Hilb }\left(\mathbb{C}^{3} / G^{\prime}\right) \longrightarrow G \text {-Hilb }\left(\mathbb{C}^{3} / G\right) \text {. }
$$

Since it is birational and since $G-\operatorname{Hilb}\left(\mathbb{C}^{3} / G\right)$ is irreducible, it must be an isomorphism.

In our main example, concerning the subgroup of index two of $\Gamma_{2}$ [2], we can avoid using this rather deep theory, since the occurring singularities are rather mild and easy to desingularize. We will describe this below.

Our main result is:

Theorem 3.7. Let be $\Gamma^{\prime}$ be any group between $\Gamma_{\mathfrak{n}}$ and $\Gamma_{0}[2]_{\mathfrak{n}}$. Then the Satake compactification of $\mathbb{H}_{2} / \Gamma^{\prime}$ admits a desingularization which is a Calabi-Yau manifold.

It remains to notice that the first Betti number vanishes. It is known that $h^{1,0}$ is zero for all non-singular models of Siegel modular varieties of genus $>1$. From Serre duality, it follows that also $h^{2,0}=0$. This fits to the computation in [12]. For $\Gamma_{2}[4]$ one has $h^{2,0}=6$. It is easy to derive from the description given in [12] that any form invariant under $\Gamma^{\prime}$, is zero.

We will give now an explicit and very simple construction of the desingularization in the case of the smallest group $\Gamma=\Gamma_{2}[2]_{\mathfrak{n}}$. We have to study the singularities of $\tilde{X}(4) / \Gamma$. For this we introduce the finite groups

$$
A=\Gamma_{2}[2] / \pm \Gamma_{2}[4] \text { and } B=\Gamma / \pm \Gamma_{2}[4]
$$


So $B$ is a subgroup of index two of $A$. The basic point now is that $A$ is an abelian group and that each element of $A$ has order $\leq 2$. Igusa [10] proved that $\tilde{X}(4) / A$ is smooth. Let $a \in \tilde{X}(4)$ be some point. Using suitable coordinates the stabilizer $A_{a}$ can be be linearized. Hence $A_{a}$ can be considered as some subgroup of GL $(3, \mathbb{C})$. Since $A_{a}$ is abelian we can diagonalize it. Hence $A_{a}$ can be considered as subgroup of $(\mathbb{Z} / 2 \mathbb{Z})^{3}$, where this group acts on $\mathbb{C}^{3}$ in the obvious way by changing signs. Since the quotient by $A_{a}$ is smooth, $A_{a}$ must be generated by reflections (here simply sign changes of one variable). Since $B$ is a subgroup of index two of $A$, we know that $B_{a}$ is a subgroup of index $\leq 2$ of $A_{a}$. Hence we obtain:

Lemma 3.8. Let $\Gamma=\Gamma_{2}[2]_{\mathfrak{n}}$. The quotient $\tilde{X}(4) / \Gamma$ looks locally like $\mathbb{C}^{3} / H$, where $H \subset(\mathbb{Z} / 2 \mathbb{Z})^{3}$ is a subgroup which is contained in some reflection subgroup as subgroup of index $\leq 2$.

The reflection subgroups of $(\mathbb{Z} / 2 \mathbb{Z})^{3}$ are trivial to describe. Up to permutation of the variables one has four cases: the trivial subgroup, sign change of the first variable, arbitrary sign changes of the first two variables and sign changes of all variables.

The group $H$ is a subgroup of index $\leq 2$ of such a group. We are only interested in cases where $H$ is not generated by reflections. There are only three types of such groups:

1. The group of order two which is generated by

$$
\left(z_{1}, z_{2}, z_{3}\right) \longmapsto\left(-z_{1},-z_{2}, z_{3}\right)
$$

2. The group of order 4 which is generated by

$$
\left(z_{1}, z_{2}, z_{3}\right) \longmapsto\left(-z_{1},-z_{2}, z_{3}\right) \text { and }\left(z_{1}, z_{2}, z_{3}\right) \longmapsto\left(z_{1}, z_{2},-z_{3}\right) .
$$

3. The group of order 4 given by sign changes of an even number of coordinates.

In the second case the group $H$ contains a reflection, changing the sign of the third variable. We can take the quotient by this reflection and thus reduce the second case to the first one. So there only two types of singularities occur, case 1 . and 3. In both cases the differential form can be written as $h(z) \mathrm{d} z_{1} \wedge \mathrm{d} z_{2} \wedge \mathrm{d} z_{3}$ with $h$ some $H$-invariant holomorphic function in a neighborhood of the origin. Since this differential form should produce a differential form without zeros on the regular locus of the quotient, we obtain that $h(0) \neq 0$. This means that we can assume that $\omega$ simply is given by $\mathrm{d} z_{1} \wedge \mathrm{d} z_{2} \wedge \mathrm{d} z_{3}$.

Now we describe the desingularization. In case 1 . we blow up $\mathbb{C}^{3}$ along the line $z_{1}=z_{2}=0$. A typical affine chart of the blow-up is given by $\left(w_{1}=z_{1} / z_{2}, z_{2}, z_{3}\right)$. Because of

$$
z_{2} \mathrm{~d} z_{1} \wedge \mathrm{d} z_{2} \wedge \mathrm{d} z_{3}=\mathrm{d} w_{1} \wedge \mathrm{d} z_{2} \wedge v z_{3}
$$

the pull-back of the differential form $\mathrm{d} z_{1} \wedge \mathrm{d} z_{2} \wedge \mathrm{d} z_{3}$ has a zero along $z_{2}=0$. The group $H$ acts now as the group generated by $\left(w_{1}, z_{2}, z_{3}\right) \mapsto\left(w_{1},-z_{2}, z_{3}\right)$. This is 
a reflection group. Hence the quotient is smooth and the zero of $\omega$ along the ramification $z_{2}=0$ disappears on the quotient, which gives the desired desingularization of $\mathbb{C}^{3} / H$.

The remaining case 3 . is slightly more involved. The group $H$ now has order 4 and consists of arbitrary sign changes of an even number of variables. The singular locus is the image of the union of the three coordinate axes in $\mathbb{C}^{3}$. This time there is no canonical way to desingularize! We have to make a choice. We choose one of the three coordinate axes, for example $z_{1}=z_{2}=0$. We start by blowing up this line. A typical affine chart of the blow up again is $\left(w_{1}=z_{1} / z_{2}, z_{2}, z_{3}\right)$. The differential form gets a zero along $z_{2}=0$. Now we have to consider the strict transform of the singular locus. It is given by $w_{1}=z_{3}=0 \mathrm{We}$ have to blow up this locus up again. A typical chart is $\left(u_{1}=w_{1} / z_{3}, z_{2}, z_{3}\right)$. The differential form $\mathrm{d} z_{1} \wedge \mathrm{d} z_{2} \wedge \mathrm{d} z_{3}$ now gets besides $z_{2}=0$ the additional zero $z_{3}=0$. The group acts on the coordinates $\left(u_{1}=z_{1} /\left(z_{2} z_{3}\right), z_{2}, z_{3}\right)$ by arbitrary sign changes of the variables $z_{2}, z_{3}$. This is a reflection group. The quotient is smooth and the zeros of the differential form disappear on the quotient.

Since in the third case we have no canonical resolution (different choices of the coordinate axes lead to resolutions which are related by flops), we have to explain how to glue the resolutions to get a resolution of the global $\tilde{X}(4) / \Gamma$. The point is that the singular locus of $\tilde{X}(4) / \Gamma$ contains itself only finitely many singular points. These points lead to the case 3 ). For each of these finitely many points one has to make a choice. But the smooth points of the singular locus lead to case 1) where we have a canonical resolution. Therefore everything fits together to a complex manifold. (This argument does not give projectivity.)

\section{Equations}

In this section we treat the case where $\mathfrak{n}$ is complementary to the standard syzygetic quadruple and $\Gamma$ means $\Gamma_{\mathfrak{n}}$ for this choice.

We give the equations for the variety $X$ in the Igusa coordinates. To simplify the notation we will write the theta constants in the form

$$
\vartheta[m]=\vartheta\left[\begin{array}{ll}
a_{1} & a_{2} \\
b_{1} & b_{2}
\end{array}\right] \text { for } m=\left(\begin{array}{l}
a_{1} \\
a_{2} \\
b_{1} \\
b_{2}
\end{array}\right)
$$

The results which we describe now can by taken from Igusa's paper (see [9, page 397]).

Theorem 4.1 (Igusa). The five modular forms

$$
y_{0}=\vartheta\left[\begin{array}{l}
00 \\
11
\end{array}\right]^{4}, \quad y_{1}=\vartheta\left[\begin{array}{l}
00 \\
01
\end{array}\right]^{4}, \quad y_{2}=\vartheta\left[\begin{array}{l}
00 \\
00
\end{array}\right]^{4},
$$




$$
y_{3}=-\vartheta\left[\begin{array}{l}
10 \\
00
\end{array}\right]^{4}-\vartheta\left[\begin{array}{l}
00 \\
11
\end{array}\right]^{4}, \quad y_{4}=-\vartheta\left[\begin{array}{l}
10 \\
01
\end{array}\right]^{4}-\vartheta\left[\begin{array}{l}
00 \\
11
\end{array}\right]^{4}
$$

generate the ring of modular forms of even weight (with trivial multipliers) for $\Gamma_{2}[2]$. The defining relation is the the quartic equation

$$
\left(y_{0} y_{1}+y_{0} y_{2}+y_{1} y_{2}-y_{3} y_{4}\right)^{2}=4 y_{0} y_{1} y_{2}\left(y_{0}+y_{1}+y_{2}+y_{3}+y_{4}\right) .
$$

Moreover according to Igusa we have the relation

$$
2 \vartheta\left[\begin{array}{l}
00 \\
01
\end{array}\right]^{2} \vartheta\left[\begin{array}{l}
00 \\
00
\end{array}\right]^{2} \vartheta\left[\begin{array}{l}
00 \\
10
\end{array}\right]^{2} \vartheta\left[\begin{array}{l}
00 \\
11
\end{array}\right]^{2}=y_{0} y_{1}+y_{0} y_{2}+y_{1} y_{2}-y_{3} y_{4} .
$$

We set

$$
y_{5}:=\Theta \quad\left(=\vartheta\left[\begin{array}{l}
00 \\
01
\end{array}\right] \vartheta\left[\begin{array}{l}
00 \\
00
\end{array}\right] \vartheta\left[\begin{array}{l}
00 \\
10
\end{array}\right] \vartheta\left[\begin{array}{l}
00 \\
11
\end{array}\right]\right) .
$$

We recall that the Calabi-Yau form $T$ is the product of the complementary six thetas. The product $y_{5} T$ is Igusa's modular form $\chi_{5}$ which is the unique modular form of weight 5 for the full modular group. The character of this form is non-trivial but trivial on $\Gamma_{2}$ [2]. Hence $y_{5}$ is a modular form of weight two with trivial character on our group $\Gamma$.

Proposition 4.2. The ring of modular forms of even weight for $\Gamma$ is generated by the six forms of weight two, $y_{0}, \ldots, y_{5}$. The defining relations are the quartic

$$
y_{5}^{4}=y_{0} y_{1} y_{2}\left(y_{0}+y_{1}+y_{2}+y_{3}+y_{4}\right)
$$

and the quadric

$$
2 y_{5}^{2}=y_{0} y_{1}+y_{0} y_{2}+y_{1} y_{2}-y_{3} y_{4} .
$$

Proof. The field of modular functions for $\Gamma$ is a quadratic extension of the field of modular functions for $\Gamma_{2}[2]$. Hence the homogenous field of fractions of $\mathbb{C}\left[y_{0}, \ldots, y_{5}\right]$ is the full field of modular functions of $\Gamma$. Since $y_{0}, \ldots, y_{5}$ have no common zero on the Satake compactification, the ring of all modular forms of even weight is the normalization of $\mathbb{C}\left[y_{1}, \ldots, y_{6}\right]$. Hence it suffices to show that the ideal given by the above two relations is a prime ideal and that the quotient is a normal ring. Since we have two relations, the factor ring is a complete intersection and hence a Cohen-Macaulay ring. Since the singular locus (as has been described in the introduction) is of codimension $\geq 2$, on can apply the well-known Serre criterion for normality.

\section{The Calabi-Yau form}

Now we use Runge's approach and consider the theta series of second kind

$$
f_{a}(Z):=\vartheta\left[\begin{array}{l}
a \\
0
\end{array}\right](2 Z)
$$


They are linked to the $\vartheta[m]$ by the classical relation

$$
\vartheta\left[\begin{array}{l}
a \\
b
\end{array}\right](Z)^{2}=\sum_{x \in\{0,1\}^{2}}(-1)^{b^{\prime} x} f_{a+x}(Z) f_{x}(Z) .
$$

Hence the rings $\mathbb{C}\left[f_{a} f_{b}\right]$ and $\mathbb{C}\left[\vartheta[m]^{2}\right]$ agree. We denote the $f_{a}$ by $f_{1}, f_{2}, f_{3}, f_{4}$ in the ordering

$$
\left(\begin{array}{l}
0 \\
0
\end{array}\right) \quad\left(\begin{array}{l}
1 \\
0
\end{array}\right) \quad\left(\begin{array}{l}
0 \\
1
\end{array}\right) \quad\left(\begin{array}{l}
1 \\
1
\end{array}\right) \text {. }
$$

The following modified version of Igusa's result Theorem 3.2 is due to Runge [15]:

Proposition 5.1. The algebra of modular forms of even weight (with trivial multipliers) with respect to the group $\Gamma_{2}$ [2] is generated by the five forms, all of weight two,

$$
\begin{aligned}
& F_{1}=f_{1}^{4}+f_{2}^{4}+f_{3}^{4}+f_{4}^{4}, \\
& F_{2}=f_{1}^{2} f_{2}^{2}+f_{3}^{2} f_{4}^{2}, \\
& F_{3}=f_{1}^{2} f_{3}^{2}+f_{2}^{2} f_{4}^{2}, \\
& F_{4}=f_{1}^{2} f_{4}^{2}+f_{2}^{2} f_{3}^{2}, \\
& F_{5}=f_{1} f_{2} f_{3} f_{4} .
\end{aligned}
$$

The defining relation is

$$
16 F_{5}^{4}=-F_{1}^{2} F_{5}^{2}+F_{1} F_{2} F_{3} F_{4}-F_{2}^{2} F_{3}^{2}-F_{2}^{2} F_{4}^{2}+4 F_{2}^{2} F_{5}^{2}-F_{3}^{2} F_{4}^{2}+4 F_{3}^{2} F_{5}^{2}+4 F_{4}^{2} F_{5}^{2} .
$$

It is very easy to describe the action of $\Gamma_{2,0}[2]$ on the generators:

Lemma 5.2. The three translations

$$
Z \longmapsto Z+S ; \quad S=\left(\begin{array}{ll}
1 & 0 \\
0 & 0
\end{array}\right),\left(\begin{array}{ll}
0 & 1 \\
0
\end{array}\right),\left(\begin{array}{ll}
0 & 0 \\
0 & 1
\end{array}\right)
$$

act by $\left(F_{1}, \ldots, F_{5}\right) \mapsto$

$$
\left(F_{1},-F_{2}, F_{3},-F_{4}, F_{5}\right), \quad\left(F_{1}, F_{2}, F_{3}, F_{4},-F_{5}\right), \quad\left(F_{1}, F_{2},-F_{3},-F_{4}, F_{5}\right) .
$$

The unimodular substitutions

$$
Z \longmapsto Z[U] ; \quad U=\left(\begin{array}{ll}
0 & 1 \\
1 & 0
\end{array}\right), \quad U=\left(\begin{array}{ll}
1 & 1 \\
0 & 1
\end{array}\right)
$$

act by

$$
\left(F_{1}, \ldots, F_{5}\right) \longmapsto\left(F_{1}, F_{3}, F_{2}, F_{4}, F_{5}\right), \quad\left(F_{1}, F_{2}, F_{4}, F_{3}, F_{5}\right) .
$$

The analogue of Proposition 4.2 is: 
Proposition 5.3. The ring of modular forms of even weight (with trivial multipliers) of $\Gamma$ is generated by six modular forms all of weight two, namely $F_{1}, \ldots, F_{5}$ (see Proposition 5.1) and the additional form $F_{6}=\Theta$. There are two defining relations, namely the relation described in Proposition 5.1 and the additional quadratic relation

$$
F_{6}^{2}=F_{1}^{2}-4 F_{2}^{2}-4 F_{3}^{2}-4 F_{4}^{2}+32 F_{5}^{2} .
$$

We know the action of $\Gamma_{2,0}$ [2] also on $F_{6}$ (Lemma 2.2). We give just one example:

Lemma 5.4. The matrix

$$
M=\left(\begin{array}{ll}
E & 0 \\
C & E
\end{array}\right) \quad C=\left(\begin{array}{ll}
2 & 2 \\
2 & 2
\end{array}\right)
$$

acts by

$$
\left(F_{1}, \ldots, F_{6}\right) \longmapsto\left(F_{1}, F_{2}, F_{3}, F_{4}, F_{5},-F_{6}\right) .
$$

In the new coordinates we compute an algebraic expression for the Calabi-Yau differential form

$$
\omega:=T \mathrm{~d} z_{0} \wedge \mathrm{d} z_{1} \wedge \mathrm{d} z_{2}, \quad Z=\left(\begin{array}{ll}
z_{0} & z_{1} \\
z_{1} & z_{2}
\end{array}\right) .
$$

As we mentioned $T y_{5}=\chi_{5}$ is the well-known cusp form of weight 5 for the full modular group. We will use the homogenous Jacobian

$$
W\left(f_{1}, f_{2}, f_{3}, f_{4}\right)=\operatorname{det}\left(\begin{array}{cccc}
f_{1} & f_{2} & f_{3} & f_{4} \\
\partial_{0} f_{1} & \partial_{0} f_{2} & \partial_{0} f_{3} & \partial_{0} f_{4} \\
\partial_{1} f_{1} & \partial_{1} f_{2} & \partial_{1} f_{3} & \partial_{1} f_{4} \\
\partial_{2} f_{1} & \partial_{2} f_{2} & \partial_{2} f_{3} & \partial_{2} f_{4}
\end{array}\right)
$$

Here $\partial_{i}$ denotes differentiation by $z_{i}$. The connection with the usual Jacobian

$$
J\left(f_{1} / f_{4}, f_{2} / f_{4}, f_{3} / f_{4}\right)=\operatorname{det}\left(\begin{array}{lll}
\partial_{0}\left(f_{1} / f_{4}\right) & \partial_{0}\left(f_{2} / f_{4}\right) & \partial_{0}\left(f_{3} / f_{4}\right) \\
\partial_{1}\left(f_{1} / f_{4}\right) & \partial_{1}\left(f_{2} / f_{4}\right) & \partial_{1}\left(f_{3} / f_{4}\right) \\
\partial_{2}\left(f_{1} / f_{4}\right) & \partial_{2}\left(f_{2} / f_{4}\right) & \partial_{2}\left(f_{3} / f_{4}\right)
\end{array}\right)
$$

is

$$
W\left(f_{1}, f_{2}, f_{3}, f_{4}\right)=f_{4}^{4} J\left(f_{1} / f_{4}, f_{2} / f_{4}, f_{3} / f_{4}\right) .
$$

The Jacobian of a modular substitution $M$ is $\operatorname{det}(C Z+D)^{-3}$. Hence $J$ is a modular form of weight 3 and $W$ is a modular form of weight 5 . If one applies a modular transformation to the $f_{i}$ one obtains a linear transformation of them. This shows that $W$ is invariant under $M$ up to the determinant of this linear transformation. This shows that $W$ up to a constant factor equals Igusa's modular form $\chi_{5}$ which is the only modular form of weight 5 for the full modular group. It can be defined as the product of the ten theta series. As a consequence we obtain

$$
d\left(f_{1} / f_{4}\right) \wedge d\left(f_{2} / f_{4}\right) \wedge d\left(f_{3} / f_{4}\right)=c \frac{\chi_{5}}{f_{4}^{4}} \mathrm{~d} z_{0} \wedge \mathrm{d} z_{1} \wedge \mathrm{d} z_{2}
$$


with a certain constant $c$. Using $T \Theta=\chi_{5}$ we get

$$
c \omega=\frac{f_{4}^{4}}{\Theta} d\left(f_{1} / f_{4}\right) \wedge d\left(f_{2} / f_{4}\right) \wedge d\left(f_{3} / f_{4}\right) .
$$

We set

$$
g_{1}=\frac{f_{1}}{f_{4}}, \quad g_{2}=\frac{f_{2}}{f_{4}}, \quad g_{3}=\frac{f_{3}}{f_{4}}
$$

and, with the notation of Proposition 5.1

$$
G_{1}=\frac{F_{2}}{F_{5}}, \quad G_{2}=\frac{F_{3}}{F_{5}}, \quad G_{3}=\frac{F_{4}}{F_{5}} .
$$

We get

$$
G_{1}=\frac{g_{1} g_{2}}{g_{3}}+\frac{g_{3}}{g_{1} g_{2}}, \quad G_{2}=\frac{g_{1} g_{3}}{g_{2}}+\frac{g_{2}}{g_{1} g_{3}}, \quad G_{3}=\frac{g_{2} g_{3}}{g_{1}}+\frac{g_{1}}{g_{2} g_{3}} .
$$

The Jacobian of this rational transformation is

$$
4 \frac{\left(g_{3}^{2}-g_{1}^{2} g_{2}^{2}\right)\left(g_{2}^{2}-g_{1}^{2} g_{3}^{2}\right)\left(g_{1}^{2}-g_{2}^{2} g_{3}^{2}\right)}{g_{1}^{4} g_{2}^{4} g_{3}^{4}}
$$

or

$$
4 \frac{\left(f_{1}^{2} f_{2}^{2}-f_{3}^{2} f_{4}^{2}\right)\left(f_{1}^{2} f_{3}^{2}-f_{2}^{2} f_{4}^{2}\right)\left(f_{1}^{2} f_{4}^{2}-f_{2}^{2} f_{3}^{2}\right)}{f_{1}^{4} f_{2}^{4} f_{3}^{4}}
$$

This gives

$$
\begin{aligned}
4 c \omega & =\frac{f_{1}^{4} f_{2}^{4} f_{3}^{4} f_{4}^{4} d\left(F_{2} / F_{5}\right) \wedge d\left(F_{3} / F_{5}\right) \wedge d\left(F_{4} / F_{5}\right)}{\Theta\left(f_{1}^{2} f_{2}^{2}-f_{3}^{2} f_{4}^{2}\right)\left(f_{1}^{2} f_{3}^{2}-f_{2}^{2} f_{4}^{2}\right)\left(f_{1}^{2} f_{4}^{2}-f_{2}^{2} f_{3}^{2}\right)} \\
& =\frac{F_{5}^{4}}{\left(F_{2} F_{3} F_{4}-2 F_{1} F_{5}^{2}\right) F_{6} d\left(F_{2} / F_{5}\right) \wedge d\left(F_{3} / F_{5}\right) \wedge d\left(F_{4} / F_{5}\right)} .
\end{aligned}
$$

The group $\Gamma_{\mathfrak{n}}$ is normal in $\Gamma_{2,0}$ [2]. The quotient acts on the ring of modular forms for $\Gamma_{\mathfrak{n}}$. The subgroup of index two

$$
G=\Gamma_{2,0}[2]_{\mathfrak{n}} / \Gamma_{2}[2]_{\mathfrak{n}}
$$

leaves the differential form invariant. Using Lemma 5.4 and Lemma 5.2 we can express the action of $G$ on the generators. We obtain:

Theorem 5.5. Let $X$ be the subvariety of $P^{5}(\mathbb{C})$ given a as intersection of the quartic

$$
16 x_{4}^{4}+x_{0}^{2} x_{4}^{2}+x_{1}^{2} x_{2}^{2}+x_{1}^{2} x_{3}^{2}+x_{2}^{2} x_{3}^{2}=x_{0} x_{1} x_{2} x_{3}+4 x_{4}^{2}\left(x_{1}^{2}+x_{2}^{2}+x_{3}^{2}\right)
$$


and the quadric

$$
x_{5}^{2}=x_{0}^{2}-4 x_{1}^{2}-4 x_{2}^{2}-4 x_{3}^{2}+32 x_{4}^{2} .
$$

This is a normal projective variety of dimension three. There exists a desingularization $\tilde{X} \rightarrow X$ which is a Calabi-Yau manifold. The differential form without zeros is given by

$$
\omega:=\frac{x_{4}^{4}}{\left(x_{1} x_{2} x_{3}-2 x_{0} x_{4}^{2}\right) x_{5}} d\left(x_{1} / x_{4}\right) \wedge d\left(x_{2} / x_{4}\right) \wedge d\left(x_{3} / x_{4}\right) .
$$

The group $G \cong S_{3} \cdot(\mathbb{Z} / 2 \mathbb{Z})^{3}$ (semidirect product) generated by

1. arbitrary permutations of $x_{1}, x_{2}, x_{3}$ followed by the sign change of $x_{5}$ if the permutation is odd,

2. arbitrary sign changes of two of the $x_{1}, x_{2}, x_{3}$,

3. the sign change of $x_{4}$,

is a group of automorphisms of $X$ which fixes $\omega$. For each subgroup $H \subset G$ the quotient X/H admits a Calabi-Yau desingularization with Calabi-Yau form $\omega$.

For sake of completeness we give the coordinate transformation between the coordinates $x_{i}$ and the coordinates $y_{i}$ which we used in the introduction. We have $y_{5}=x_{5}$ and

$$
\left(\begin{array}{l}
y_{0} \\
y_{1} \\
y_{2} \\
y_{3} \\
y_{4}
\end{array}\right)=\left(\begin{array}{rrrrr}
1 & -2 & -2 & 2 & 0 \\
-2 & 2 & -2 & 0 \\
2 & 2 & 2 & 0 \\
-1 & 2 & -2 & -2 & -8 \\
-1 & 2 & -2 & -2 & 8
\end{array}\right)\left(\begin{array}{l}
x_{0} \\
x_{1} \\
x_{2} \\
x_{3} \\
x_{4}
\end{array}\right) .
$$

The expressions we obtained for $\omega$ and for the action of $G$ in the coordinates $y_{i}$ looked not very nice, hence we skip them.

\section{References}

[1] W. BARTH and I. NIETO, Abelian surfaces of type $(1,3)$ and quartic surfaces with 16 skew lines, J. Algebraic Geom. 3 (1994), 173-222.

[2] T. BRIDGELAND, A. KING and M. REID, The McKay correspondence as an equivalence of derived categories, J. Amer. Math. Soc. 14 (2001), 535-554.

[3] E. Freitag, "Siegelsche Modulfunktionen", Grundlehren der mathematischen Wissenschaften, Bd., Vol. 254, Berlin Heidelberg New York: Springer, 1983.

[4] V. GRITSENKO and F. CLÉRY, The Siegel modular form of genus 2 with the simplest divisor, arXiv: 0812.3962 (2008).

[5] V. GRitsenko and K. Hulek, The modular form of the Barth-Nieto quintic, Internat. Math. Res. Notices 17 (1999).

[6] B. VAN GEEMEN and N. O. NyGAARD, On the geometry and arithmetic of some Siegel modular threefolds, J. Number Theory 53 (1995), 45-87. 
[7] B. Van Geemen and D. van Straten, The cusp form of weight 3 on $\Gamma_{2}(2,4,8)$, Math. Comp. 61 (1993), 849-872.

[8] B. van Geemen, K. Hulek, J. Spandau and D. van Straten, The modularity of the Barth-Nieto quintic and its relatives, Adv. Geom. 1 (2001), 263-289.

[9] I. IGUS A, On Siegel modular forms of genus two II, Amer. J. Math. 86 (1964), 392-412.

[10] I. IgUSA, A desingularization problem in the theory of Siegel modular functions, Math. Ann. 168 (1967), 228-260.

[11] Y. ITO, Gorenstein quotient singularities of monomial type in dimension three, J. Math. Sci. Univ. Tokyo 2 (1995), 419-440.

[12] R. LEE, and S. H. Weintraub, The Siegel modular variety of degree two and level four, Mem. Amer. Math. Soc. 631 (1998), 3-58.

[13] M. REID, Minimal models of canonical 3-folds, In: "Algebraic Varieties and Analytic Varieties" (Tokyo, 1981), Adv. Stud. Pure Math., Vol. 1, Amsterdam: North-Holland, 131-180.

[14] M. REID, "La correspondence de McKay", Séminaire Bourbaki 1999/2000, Astérisque, Vol. 276, 2002, 53-72.

[15] B. Runge, On Siegel modular form, part I, J. Reine Angew. Math. 436 (1993), 57-85.

[16] B. Runge, On Siegel modular forms, part II, Nagoya Math. J. 138 (1995), 179-197.

[17] R. Salvati ManNi, Thetanullwerte and stable modular forms, Amer. J. Math. 111 (1989), 435-455.

\author{
Mathematisches Institut \\ Universität Heidelberg \\ Im Neuenheimer Feld 288 \\ 69120 Heidelberg \\ freitag@mathi.uni-heidelberg.de \\ Dipartimento di Matematica \\ Sapoienza Università di Roma \\ Piazzale Aldo Moro, 5 \\ 00185 Roma, Italia \\ salvati@mat.uniroma1.it
}

